\title{
Laparoscopic Excision of a Retroperitoneal Solitary Fibrous Tumor: A Case Report
}

\author{
Eun-Ki Min, B.S. ${ }^{1}$, Younghae Song, M.D. ${ }^{2}$, Jae Uk Chong, M.D. ${ }^{3,4}$, Chang Moo Kang, M.D., Ph.D. ${ }^{3,4}$ \\ ${ }^{1}$ Yonsei University College of Medicine, ${ }^{2}$ Korea Medical Institute, ${ }^{3}$ Department of Hepatobiliary and Pancreatic Surgery, Department of Surgery, Yonsei \\ University College of Medicine, ${ }^{4}$ Pancreatobiliary Cancer Clinic, Yonsei Cancer Center, Severance Hospital, Seoul, Korea
}

Solitary fibrous tumors (SFTs) are rare mesenchymal tumors mainly originating in the pleura. Since complete resection is the most important prognostic factor, typical surgical approach has been open laparotomy. In this report, we present a unusual case of large retropancreatic SFT that was successfully treated via laparoscopic resection. A 22-year-old female was diagnosed with a $8 \times 7$ $\mathrm{cm}$-sized well-demarcated mass with multiple loculating and enhancing solid portions on the left adrenal fossa. The mass showed no definite invasion of adjacent organs and laparoscopic resection was planned. Using blunt dissection and individual vessel ligation, the operation was successful. The operative time was 220 minutes, and the amount of intraoperative blood loss was estimated to be within $100 \mathrm{ml}$. The patient recovered without complications. Laparoscopic excision of large retroperitoneal SFTs can be safe and feasible if there is no evidence of local invasion or malignancy on preoperative radiologic images.

Keywords: Minimally invasive surgical procedures, Solitary fibrous tumors, Retroperitoneal neoplasms

This is an Open Access article distributed under the terms of the Creative Commons Attribution Non-Commercial License (http:// creativecommons.org/licenses/by-nc/4.0/) which permits unrestricted non-commercial use, distribution, and reproduction in any medium, provided the original work is properly cited.
Received June 13, 2016

Revised August 31, 2016

Accepted September 2, 2016

Corresponding author

Chang Moo Kang

Department of Hepatobiliary and Pancreatic Surgery, Yonsei University College of Medicine, Seongsanno 250, Seodaemun-gu, Seoul 03722, Korea

Tel: +82-2-2228-2135

Fax: +82-2-313-8289

E-mail: cmkang@yuhs.ac

Copyright ( $) 2016$ The Journal of Minimally Invasive Surgery. All rights reserved.

\section{INTRODUCTION}

Solitary fibrous tumor (SFT) is a rare mesenchymal neoplasm initially documented as arising from the pleura. ${ }^{1}$ SFTs commonly involve the pleura and abdominopelvic cavity, but they can occur in almost every anatomic site and organ. ${ }^{2}$ Nearly 30\% of SFT has been reported to arise from extrapleural locations and SFTs arising in the retroperitoneum are rare with only a few cases reported. ${ }^{3}$ The majority of SFTs are regarded as benign and only 12 22\% are histologically or clinically malignant. ${ }^{4}$ Typical SFTs show sporadic or no mitotic figures with mild nuclear atypia. Malignant SFT is defined on the basis of infiltrative margins, hypercellularity, nuclear atypia, mitotic index greater than 4/10 high-power fields, and necrosis. ${ }^{5}$ Most SFTs are asymptomatic and present as a pain- less mass. However, rare and unspecific symptoms may present depending on location, most commonly as a local pressure effect. Surgical excision is the mainstay of treatment, and complete resectability is the most important prognostic factor. ${ }^{5}$ For that reason, the surgical approach has typically been open laparotomy for reported cases of retroperitoneal SFT. ${ }^{3}$

Early in the development of laparoscopic surgery, application to retroperitoneal tumors was limited due to technical difficulties resulting from the deep location and close proximity to major vessels. With recent advances in laparoscopic surgical techniques in the hands of experienced surgeons, successful laparoscopic approaches to various retroperitoneal tumors, including cystic lymphangioma, schwannoma, ganglioneuroma, and liposarcoma, have been reported. In this report, we present a case of a young female patient with a large 
retropancreatic SFT that was successfully treated via laparoscopic resection.

\section{CASE REPORT}

\section{Patient}

A 22-year-old female patient was referred to our institution for an asymptomatic incidental large retropancreatic mass that was found during a routine medical check-up. The patient's past medical history and family history were unremarkable. On physical examination, her abdomen was soft and nontender without a palpable mass. Initial abdominal ultrasound has revealed a $8 \times 7 \mathrm{~cm}$ sized well-demarcated and multiseprated mass on pancreas tail. The mass was in close proximity to liver, spleen, and left kidney but borders were well-defined without invasion. Initial impression was a cystic neoplasm of pancreas tail and further evaluation with abdominal computed tomography (CT) and magnetic resonance imaging (MRI) scans were performed. The results demonstrated a $8 \times 7 \mathrm{~cm}$ sized well-demarcated mass with multiple loculating and enhancing solid portions on the left adrenal fossa under the left side of the pancreas (Fig. 1). Impression after further imaging study was a retroperitoneal tumor in left adrenal fossa with a differential diagnosis needed for solitary fibrous tumor, gastrointestinal stromal tumor, schwannoma, pleomorphic lipoma and mucinous cystadenoma. The mass showed a relatively smooth margin without definite invasion of adjacent organs. Therefore, laparoscopic resection was planned.

\section{Surgery}

Under general endotracheal anesthesia, the patient was placed on the surgical table in the supine position. The patient's head and left side were elevated. A $1.5^{-} \mathrm{cm}$ skin incision was made over the umbilicus and the peritoneum was opened using the open method approach. A 12-mm trocar was inserted and $\mathrm{CO}_{2}$ gas was insufflated through the trocar at a speed of $20 \mathrm{~L} / \mathrm{min}$ and up to the intraperitoneal pressure of $12 \mathrm{mmHg}$. After inserting the telescope, positions for two working ports on each side of subcostal area were determined. Right subcostal port was placed on about $2 \mathrm{~cm}$ right side of the falciform ligament and left subcostal port was appropriately placed to facilitate easy access to left adrenal fossa. Upon laparoscopic examination under the pneumoperitoneum, an $8 \mathrm{~cm}$ solid mass was noted between the kidney, stomach, pancreas, and spleen. After division of the gastrocolic ligament, the tumor was carefully dissected from the surrounding tissue and there was no macroscopic evidence of malignancy; thus, the decision was made to continue the laparoscopic approach. Since the tumor did not show invasion to the surrounding tissues or organs, careful and blunt dissection around the tumor
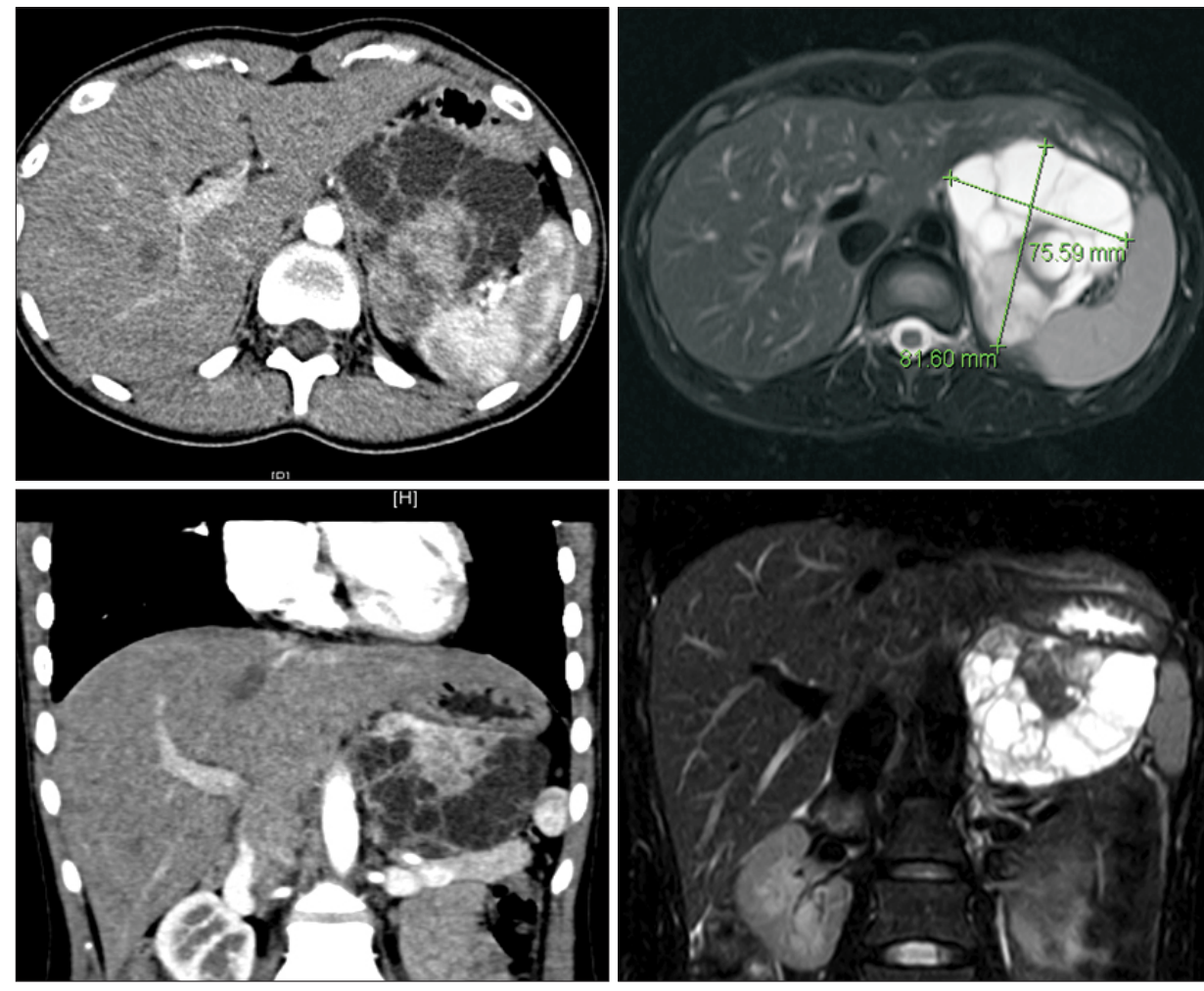

Fig. 1. Preoperative imaging studies. Contrast-enhanced abdominal computed tomography and magnetic resonance imaging revealed a $8.4 \times 7.5-\mathrm{cm}$ mass on the left adrenal fossa with internal multiple loculations and an enhancing solid portion. The mass had a relatively smooth margin without definite invasion of adjacent organs. 
enabled isolation of feeding vessels. The several feeding vessels that form the celiac axis and splenic hilum were all safely controlled and divided (Fig. 2). The tumor was safely delivered by a small vertical extension of the umbilical port site. One silastic drain was placed into the surgical field. The operative time was 220 minutes, and the amount of intraoperative blood loss was estimated to be within $100 \mathrm{ml}$ during the whole laparoscopic procedure. The patient recovered without complications. Oral diet was started immediately and patient was discharged on the fifth day after the surgery. The patient was followed up for more than 5 years after the surgery without evidence of local or systemic recurrence.

\section{Pathological findings}

Grossly, the resected mass weighed $150 \mathrm{~g}$ and measured $8 \times$ $6 \times 6.5 \mathrm{~cm}$. Inside the mass, there were multilocular cysts $(3.3 \times$ $3 \mathrm{~cm}$ in the largest one) with a solid area $(4.5 \times 4 \mathrm{~cm})$. The solid area was homogeneously grayish pink and soft. On further sections, the cyst contained clear fluid and the inner wall of the cyst was smooth. The pathologic examination proved that the tumor was a solitary fibrous tumor with diffuse positivity
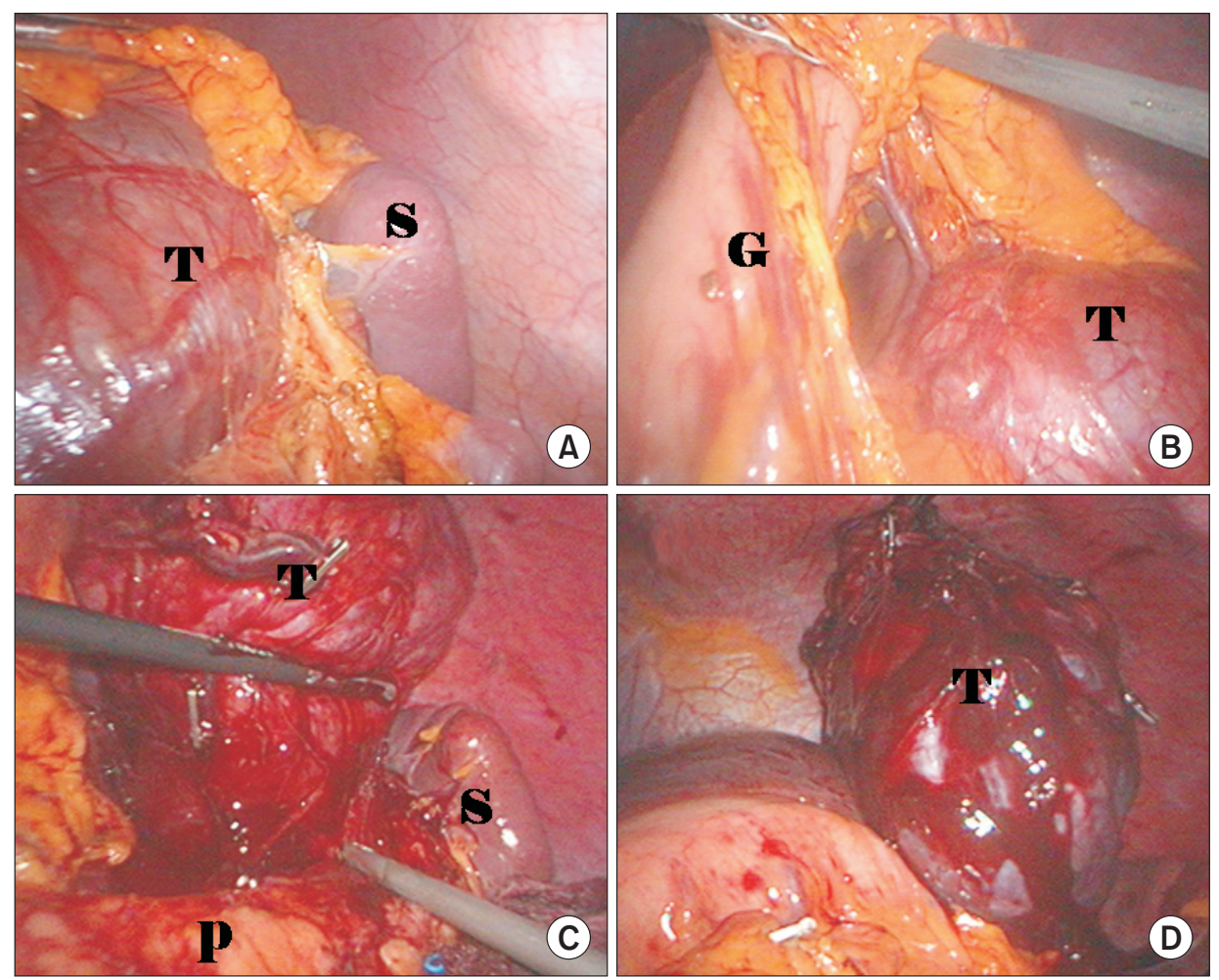

Fig. 2. Intraoperative laparoscopic views. (A) The tumor was identified between the kidney, stomach, pancreas and spleen. (B, C) Several feeding vessels were noted and safely ligated. (D) The successfully resected retroperitoneal mass. $T=$ tumor; $S=$ spleen; $P=$ pancreas; $G=$ greater curvature of the stomach.
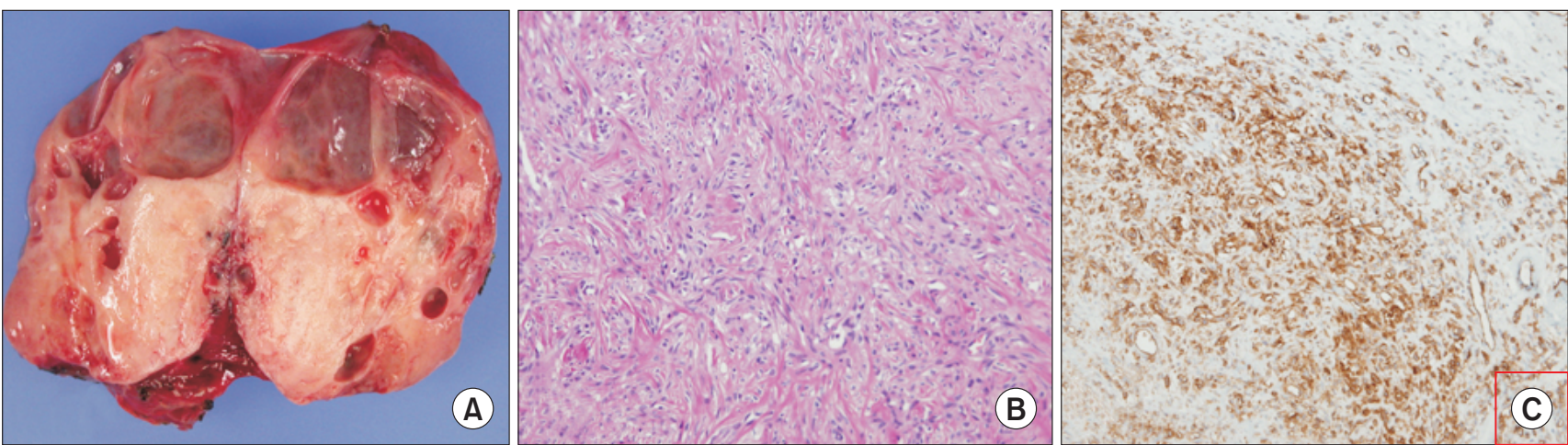

Fig. 3. Pathologic examination: (A) Gross specimen with multilocular cysts and a solid portion. (B) Histological examination of SFT. The tumor consisted mainly of spindle-shaped cells (H\&E stain, $\times 100$ ). (C) Immunohistochemical findings of SFT. The tumor cells showed immunoreactivity to CD34 diffusely in their cytoplasm. 
for CD 34 and focal positivity for S-100 protein on immunohistochemical stain (Fig. 3).

\section{DISCUSSION}

Since its first documentation in 1931, ${ }^{1}$ SFT has long been regarded as a rare pleural neoplasm. It is currently believed to originate from mesenchymal fibroblast-like cells ${ }^{6}$ and has been shown to involve nearly all body sites. ${ }^{2}$ SFT has a peak incidence in middle-aged adults (range, 20 70 years) and affects both sexes equally. ${ }^{7}$ SFT usually presents as a slowgrowing painless mass or may compress adjacent structures causing symptoms like abdominal pain. Although abdominopelvic CT and MRI of a large, solid, well-defined, hypervascular mass with necrosis or cystic degeneration could raise a preoperative suspicion of SFT, confirmation of the diagnosis still depends on histological and immunohistochemical examination.

Histologically, the characteristic features of SFT are a patternless distribution of bland ovoid or spindled fibroblastic cells with intercellular dense collagen and interspersed large branching thin-walled hemangiopericytic vessels. ${ }^{6}$ Malignant SFT is defined on the basis of infiltrative margins, hypercellularity, nuclear atypia, mitotic index greater than 4/10 highpower fields, and necrosis. ${ }^{5}$ Immunohistochemically, several proteins such as CD34, vimentin, B cell lymphoma 2 (BCL-2), and $\mathrm{CD} 99$, are generally considered to have diagnostic value for SFT. ${ }^{8}$ Recently, STAT6 has been reported as a promising marker, expression of which is predominantly nuclear in SFT. ${ }^{9}$ CD34, a positive marker for SFT, is expressed on the surface of lymphohematopoietic stem and progenitor cells, small-vessel endothelial cells, and embryonic fibroblasts. ${ }^{3}$ SFT typically shows diffuse and strong expression of CD34, as well as CD99. Positive finding for vimentin, a general marker of cells originating in the mesenchyme, also support the diagnosis of SFT. And $\mathrm{Bcl}-2$, which suppresses apoptosis, has been reported to show positive reactivity in $75 \%$ of extrapleural SFT. ${ }^{3}$ In our case, diffusely positive CD34 was found on pathology, thus establishing a diagnosis of SFT. CD31, S-100 protein, desmin, and cytokeratins are usually absent in SFT. Nevertheless, some focal expression of those markers has been reported, ${ }^{10}$ including our case of focal positivity for S-100.

The differential diagnosis of SFT includes schwannoma, spindle cell/pleomorphic lipoma, dermatofibrousarcoma protuberans, gastrointestinal stromal tumors, dedifferentiated liposarcoma, well-differentiated liposarcoma, and synovial sarcoma; immunohistochemical staining and cytogenetic features can be used for differentiation.

SFT has been reported to show unpredictable behavior; even SFTs without malignant histologic features can abruptly dedifferentiate into aggressive soft tissue sarcoma. Therefore, long-term follow-up is required. According to van Houdt et al., ${ }^{8} 5$-year local recurrence and metastasis rate after the operation with curative intent were 29 and 34\%, respectively. The 5 -year overall survival was reported to be $84 \%$. While SFT is generally regarded as benign, substantial amount of patients develop recurrence or metastasis. Therefore, long-term follow up period is necessary. Among the factors related to recurrence and metastasis, positive resection margin has been associated with a higher local recurrence rate, whereas large tumor size $(>10 \mathrm{~cm})$ and high mitosis rate $(>4$ per $10 \mathrm{HPFs})$ correlated with higher metastasis rate.

Wide surgical excision is the standard treatment of SFTs, and margin negative resection is the most important prognostic factor, in view of its unpredictable nature. ${ }^{8}$ Retroperitoneal SFTs are rare and traditionally have been treated via open laparotomy rather than laparoscopic surgery to ensure margin-negative, and safe resection. With recent advances in laparoscopic technique, along with improved imaging modalities, laparoscopic resection of retroperitoneal tumors has become feasible, even in cases of large tumors in difficult locations such as close to the vena cava or aorta. Herein, we presented a case of large retroperitoneal SFT treated with laparoscopic resection. In spite of its large size and the need for ligation of feeding vessels from the celiac axis and splenic hilum, laparoscopic excision was feasible and safe, with minimal invasiveness, early postoperative recovery and cosmetic benefits. Successful laparoscopic excision was made possible, because SFT had no local invasion to surrounding tissue with margins well-demarcated. Careful blunt dissection around the tumor allowed isolation of feeding vessels and individual ligation of vessels. The quality of life of the young female patient was not compromised and there has been no evidence of local or systemic recurrence in the 5 years after surgery.

In conclusion, laparoscopic excision of large retroperitoneal SFTs can be safe and feasible if there is no evidence of local invasion or malignancy on preoperative radiologic images. However, adequate long-term follow-up is necessary due to the possibility of late recurrence.

\section{REFERENCES}

1) Klemperer $P$, Coleman BR. Primary neoplasms of the pleura. $A$ report of five cases. Am J Ind Med 1992;22:1-31.

2) Demicco EG, Park MS, Araujo DM, et al. Solitary fibrous tumor: a clinicopathological study of 110 cases and proposed risk assessment model. Mod Pathol 2012;25:1298-1306.

3) Nomura T, Satoh R, Kashima K, et al. A case of large solitary fibrous tumor in the retroperitoneum. Clin Med Case Rep 2009;2: 21-25. 
4) Robinson LA. Solitary fibrous tumor of the pleura. Cancer Control 2006;13:264-269.

5) England DM, Hochholzer L, McCarthy MJ. Localized benign and malignant fibrous tumors of the pleura. A clinicopathologic review of 223 cases. Am J Surg Pathol 1989;13:640-658.

6) Ali SZ, Hoon V, Hoda S, Heelan R, Zakowski MF. Solitary fibrous tumor. A cytologic-histologic study with clinical, radiologic, and immunohistochemical correlations. Cancer 1997;81:116-121.

7) Fletcher $\mathrm{CD}$. The evolving classification of soft tissue tumours - an update based on the new 2013 WHO classification. Histopa- thology 2014;64:2-11.

8) van Houdt WJ, Westerveld CM, Vrijenhoek JE, et al. Prognosis of solitary fibrous tumors: a multicenter study. Ann Surg Oncol 2013; 20:4090-4095.

9) Demicco EG, Harms PW, Patel RM, et al. Extensive survey of STAT6 expression in a large series of mesenchymal tumors. Am J Clin Pathol 2015;143:672-682.

10) Hanau CA, Miettinen M. Solitary fibrous tumor: histological and immunohistochemical spectrum of benign and malignant variants presenting at different sites. Hum Pathol 1995;26:440-449. 